\title{
Invertase Suc2-mediated inulin catabolism is regulated at the transcript level in Saccharomyces cerevisiae
}

\author{
Fan Yang, Zhi-Cheng Liư ${ }^{\dagger}$ Xue Wang ${ }^{\dagger}$, Li-Li Li, Lan Yang, Wen-Zhu Tang, Zhi-Min Yu and Xianzhen Li
}

\begin{abstract}
Background: Invertase Suc2 was recently identified as a key hydrolase for inulin catabolism in Saccharomyces cerevisiae, whereas the Suc2 activity degrading inulin varies greatly in different $S$. cerevisiae strains. The molecular mechanism causing such variation remained obscure. The aim of this study is to investigate how Suc2 activity is regulated in S. cerevisiae.
\end{abstract}

Results: The effect of SUC2 expression level on inulin hydrolysis was investigated by introducing different SUC2 genes or their corresponding promoters in S. cerevisiae strain BY4741 that can only weakly catabolize inulin. Both inulinase and invertase activities were increased with the rising SUC2 expression level. Variation in the promoter sequence has an obvious effect on the transcript level of the SUC2 gene. It was also found that the high expression level of SUC2 was beneficial to inulin degradation and ethanol yield.

Conclusions: Suc2-mediated inulin catabolism is regulated at transcript level in S. cerevisiae. Our work should be valuable for engineering advanced yeast strains in application of inulin for ethanol production.

Keywords: Invertase, Transcript level, Saccharomyces cerevisiae, Inulin

\section{Background}

Inulin is a natural storage carbohydrate consisting of a linear $\beta$-2,1-linked $\mathrm{D}$-fructofuranose chain terminated by a glucose residue through a sucrose-type linkage at the reducing end [1]. As an important material for biofuel such as ethanol production, inulin produced by Jerusalem artichoke has many advantages over other feedstock, e.g., cultivation of Jerusalem artichoke does not occupy farmland and Jerusalem artichoke has strong resistance to plant disease $[2,3]$.

Although some yeast strains including Kluyveromyces, Candida and Schizosaccharomyces could directly ferment inulin without pretreatment, they are not so efficient for ethanol production [3-5]. Saccharomyces cerevisiae is the kind of efficient organism in regard of large-scale ethanol production, whereas most strains can not ferment large polymers of inulin [6]. Fortunately, several specific $S$. cerevisiae strains have been

\footnotetext{
* Correspondence: xianzhen@mail.com

${ }^{\dagger}$ Equal contributors

School of Biological Engineering, Dalian Polytechnic University, Dalian 116034, PR China
}

reported to have an ability to convert inulin-type sugars into ethanol without additional hydrolysis pretreatment [7-9]. Thus, here comes a question: what makes those inulin-degrading strains distinct from the others? Recently, the enzymatic hydrolysis of inulin in inulin-fermenting S. cerevisiae strain JZ1C was studied by Wang et al. [10]. An invertase Suc2, which was considered as a sucrose hydrolyzing enzyme, was purified and proved to be a key component able to degrade inulin for ethanol production in S. cerevisiae strain JZ1C [10]. It was also found that the amino acid sequence variation in Suc2 of strain JZ1C compared with that of weak-inulin-degrading strain S288c did not result in the change of specific enzyme activity [10]. Those results present the most possible explanation that the variation of SUC2 transcription determines the inulin utilization traits of different $S$. cerevisiae strains.

SUC2 transcript level in S. cerevisiae strains with different inulin-degrading activities was investigated in this study. Effect of transcription on the capability of catabolizing inulin in S. cerevisiae was determined by tuning 
the expression level of SUC2 with different strength promoter. Our work aims to reveal the influence of the transcript level on Suc2 activity and the regulation of SUC2 expression.

\section{Results}

Invertase Suc2 in different wild-type S. cerevisiae strains

As shown in Figure 1, the difference in ethanol production from inulin was observed in S. cerevisiae strain BY4741, NCYC625 and L610. Strain BY4741 produced low ethanol of $16 \mathrm{~g} / \mathrm{L}$, while strain NCYC625 produced higher ethanol of $38 \mathrm{~g} / \mathrm{L}$ and strain $\mathrm{L} 610$ produced the highest ethanol of $58 \mathrm{~g} / \mathrm{L}$. The invertase and inulinase activities were well correlated with the ethanol productivity (Figure 1), suggesting that the high invertase activity was essential for ethanol production from inulin in S. cerevisiae. There was no considerable difference in the cell growth among those wild-type strains when incubated in the inulin medium. It was presumed that the great variation in invertase activity among those strains was attributed to the differences in $S U C 2$ genes or their corresponding regulation. Therefore, the cause for discrepancy in inulin utilization was investigated.

\section{Homologous SUC2 ORFs encoding sucrose hydrolase had no difference in inulin-degrading activities}

The previous report showed that the invertase Suc2 was responsible for the inulin degradation in S. cerevisiae [10], thus it was proposed that the Suc2 activities were largely varied in S. cerevisiae strains with different ability of inulin utilization. Such discrepancy in Suc2 activity may be attributed to the single nucleotide polymorphisms (SNPs) of encoding sequences [11] or the varied transcription level caused by difference of promoter strength [12].

The effect of SNPs on SUC2 expression was evaluated as well as the performance of Suc2 in degrading inulin. The invertase encoding ORF SUC2 was cloned from S. cerevisiae strains NCYC625 and L610. Their nucleotide sequences were compared with the reported sequences from S. cerevisiae strains BY4741 and strain JZ1C (Figure 2). Gene alignment showed that $S U C 2$ gene from strain L610 shared the same sequence with that from strain JZ1C (data not shown). Differences in amino acid sequence were observed in Suc2 from strains L610, NCYC625 and BY4741, whereas no sequence substitution in strains L610, NCYC625 and BY4741 was located at the substrate binding domain, catalytic domain or glycosylation site [13-15] (Figure 2).

In order to determine their performance for inulin hydrolysis, three different SUC2 versions from strains L610 (LS), NCYC625 (NS) and BY4741 (BS) were expressed respectively in the weak-inulin-utilizing strains BY4741 and NCYC625. All the constructs were cultured in the inulin medium, and cell growth, ethanol production and enzyme activity were assayed. The activities of invertase and inulinase in BY4741 recombinants were comparable with that in strain L610 when different versions of SUC2 gene were overexpressed in strain BY4741 under the control of constitutive PGK1 promoter (Figure 3A). The increased ethanol production was also obtained in BY4741 recombinants, which was much higher than that in the control strain BY4741 (Figure 3A). However, the ethanol production by three recombinants was still lower than that by strain L610. The ion chromatography of the culture supernatant showed that recombinants BY4741-BS, BY4741-NS and BY4741-LS could efficiently ferment inulin with degree of polymerization less than 20 , compared with the control strain BY4741 that can only assimilate glucose, fructose and sucrose (Figure 3B). The inulin-degrading activity and preference of BY4741 recombinants was comparable with that of the strain L610. Moreover, no obvious difference was observed in the inulin utilization profile when SUC2 was overexpressed

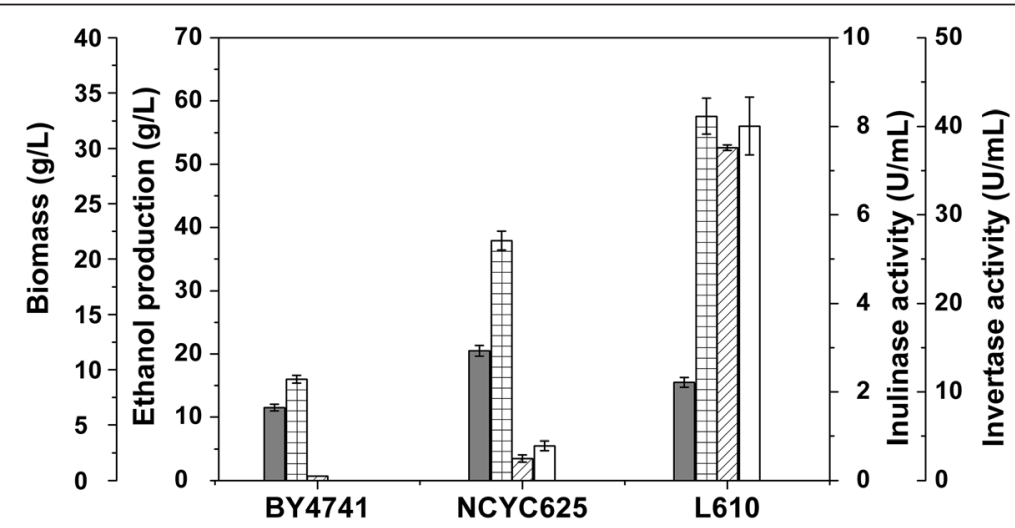

Figure 1 Inulin fermentation results of different wild-type yeast strains. Biomass (dry cell weight, gray), ethanol production (grid), inulinase activity (diagonal) and invertase activity (blank) were measured when S. cerevisiae strains BY4741, NCYC625 and L610 were cultured using inulin as a carbon source for $48 \mathrm{~h}$. Error bars represent standard deviations from the mean of three biological samples. 
MLLQAFLFLLAGFAAKISASMTNETSDRPLVHFT PNKGWMNDPNGLWYDEKDAKWHLYFQY

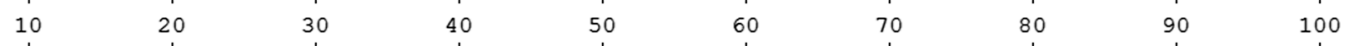

MLLQAFLFLLAGFAAKISASMTNETSDRPLVHFTPNK

MLLQAFLFLLAGFAAKISASMTNETSDRPLVHFT PNK WW WNDPNGLWYDEKDAKWHLYFQYNPNDTVWGTPLFWGHATSDDLTHWEDEPIAIAPKRNDSG NS MLLQAFLFLLAGFAAKISASMTNETSDRPLVHFT PNK WWMNDPNGLWYDEKDAKWH L YFQYNPNDTVWGT PLFWGHATSDDLTHWEDEPIAIAPKRNDSG JS MLLQAFLFLLAGFAARISASMTNETSDRPLVHFT PNKGWWNDPNGLWYDEKDAKWHLYFQYNPNDTVWGT PLFWGHATSDDLTHWEDEPIAIAPKRNDSG LS AFSGSMVVDYNNTSGFFNDTIDPRQRCVAIWTYNTPESEEQYISYSLDGGYTFTEYQKNPVLAANSTQFRDPKVFWYEPSQKWIMTAAKSQDYKIEIYSS MajOritY

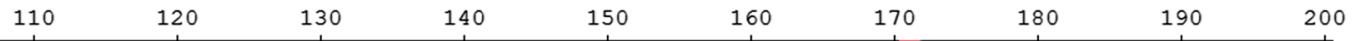

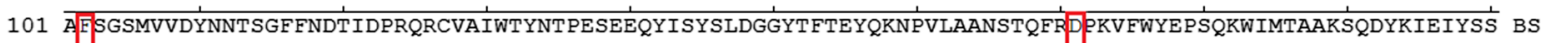

101 AFSGSMVVDYNNTSGFFNDTIDPRQRCVAIWTYNTPESEEQYISYSLDGGYTFTEYQKNPVLAANSTQFADEKVFWYEPSQKWIMTAAKSQDYKIEIYSS NS

101 A SGSMVVDYNNTSGFFNDTIDPRQRCVAIWTYNTPESEEQYISYSLDGGYTFTEYQKNPVLAANSTQFPDEKVFWYEPSQKWIMTAAKSQDYKIEIYSS JS

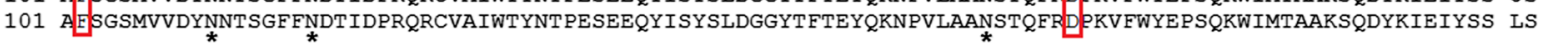

DDLKSWKLESAFANEGFLGYQYECPGLIEVPTEQDPSKSYWVMFISINPGAPAGGSFNQYFVGSFNGTHFEAFDNQSRVVDFGKDYYALQTFFNTDPTYG MajOritY

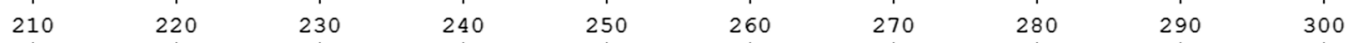

201 DDLKSWKLESAFANEGFLGYQY ECPGLIEVPTEQDPSKSYWVMFISINPGAPAGGSFNQYFVGSFNGTHFEAFDNQSRVVDFGKYYALQTFFNTDPTYG BS 201 DDLKSWKLESAFANEGFLGYQY E PGLIEVPTEQDPSKSYWVMFISSNPGAPAGGSFNQYFVGSFNGTHFEAFDNQSRVVDFGKDYYALQTFFNTDPTYG NS 201 DDLKSWKLESAFANEGFLGYQYECPGLIEVPTEQDPSKSYWVMFISINPGAPAGGSFNQYFVGSFNGTHFEAFDNQSRVVDFGKDYYALQTFFNTDPTYG JS

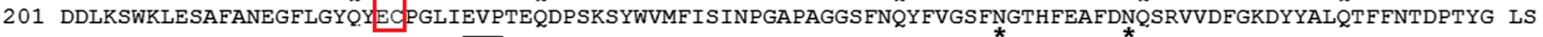

SALGIAWASNWEYSAFVPTNPWRSSMSLVRKFSLNTEYQANPETELINLKAEPILNISNAGPWSRFATNTTLTKANSYNVDLSNSTGTLEFELVYAVNTT MajOritY

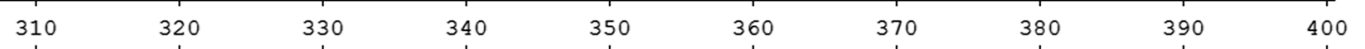

301 SALGIAWASNWEYSAFVPTNPWRSSMSLVRKFSLNTEYQANPETELINLKAEPILNISNAGPWSRFATNTTLTKANSYNVDLSNSTGTLEFELVYAVNTT BS 301 SALGIAWASNWEYSAFVPTNPWRSSMSLVRKFSLNTEYQANPETELINLKAEPILNISNAGPWSRFATNTTLTKANSYNVDLSNSTGTLEFELVYAVNTT NS 301 SALGIAWASNWEYSAFVPTNPWRSSMSLVRKFSLNTEYQANPETELINLKAEPILNISNAGPWSRFATNTTLTKANSYNVDLSNSTGTLEFELVYAVNTT JS

301 SALGIAWASNWEYSAFVPTNPWRSSMSLVRKFSLNTEYQANPETELINLKAEPILNISNAGPWSRFATNTTLTRANSYNVDLSNSTGTLEFELVYAVNTT LS

QTISKSVFPDLSLWFKGLEDPEEYLRMGFEASASSFFLDRGNSKVKFVKENPYFTNRMSVNNQPFKSENDLSYYKVYGLLDQNILELYFNDGDVVSTNTY MajOritY

$\begin{array}{lllllllll}410 & 420 & 430 & 440 & 450 & 460 & 470 & 480 & 490\end{array}$

401 QTISKSVFADDLSLWFKGLEDPEEYLRMGFEVSASSFFLDRGNSKVKFVKENPYFTNRMSVNNQPFKSENDLSYYKVYGLIDQNILELYFNDGDVVSTNTY BS

401 QTISKSVFPDLSLWFKGLEDPEEYLRMGFEVSASSFFLDRGNSKVKFVKENPYFTNRMSVNNQPFKSENDLSYYKVYGLLDQNILELYFNDGDVVSTNTY NS

401 QTISKSVFPDLSLWFKGLEDPEEYLRMGFEASASSFFLDRGNSKVKFVKENPYFTNRMSVNNQPFKSENDLSYYKVYGLLDQNILELYFNDGDVVSTNTY JS

401 QTISKSVFPDLSLWFKGLEDPEEYLRMGFEASASSFFLDRGNSKVRFVKENPYFTNRMSVNNQPFKSENDLSYYKVYGLIDQNILELYFNDGDVVSTNTY LS

FMTTGNALGSVNMTTGVDNLFYIDKFQVREVK-

Majority

$510 \quad 520 \quad 530$

$501 \overline{\text { FMTTGNALGSVNMTTGVDNLFYIDKFQVREVK }}$ BS

501 FMTTGNALGSVNMTTGVDNLFYIDKFQVREVK NS

501 FMTTGNALGSVNMTTGVDNLFYIDKFQVREVK JS

501 FMTTGNALGSVNMTTGVDNLFYIDKFQVREVK LS

Figure 2 Amino acid sequence alignment of Suc2 from different S. cerevisiae strains. Amino acid variation in Suc2 from different S. cerevisiae strains BY4741 (BS), NCYC625 (NS), JZ1C (JS) and L610 (LS). Variable amino acid residues after sequence alignment were highlighted in black. "**" refers to N-glycosylation site. Substrate binding/catalysis sites were red boxed. High conserved regions were underlined.

in recombinants BY4741 with different SUC2 genes (Figure 3B).

Similarly, overexpression of different SUC2 versions in strain NCYC625 also resulted in a dramatic increase in both enzyme activity and ethanol production from inulin (Figure 3C). Those results demonstrated that the constitutive overexpression of SUC2 in the weak-inulinutilizing strain endowed it with high ethanol production from inulin. The similar performance of three different SUC2 versions suggested that the variation of sucrose hydrolase was not attributed to the SNPs of encoding sequences. Therefore, the transcription level of SUC2 is a potential determinant of inulin utilization in S. cerevisiae.

\section{SUC2 transcription level determines the activity of inulin degradation}

In order to investigate the relationship between SUC2 expression level and inulin utilization, the mRNA expression of SUC2 was assayed by qRT-PCR in $S$. cerevisiae strains BY4741, NCYC625 and L610, which showed different inulin-degrading activity. As shown in Figure 4A, the SUC2 transcript in inulin-utilizing strain L610 was much higher than that in weak-inulin-utilizing strains NCYC625 and BY4741. Such discrepancy of SUC2 expression level in those three strains was in accordance with that of the invertase activity (Figure 1). Therefore, high expression of SUC2 is essential for the inulin degradation in $S$. cerevisiae, and low expression of SUC2 in weak-inulin-utilizing strain is presumably due to the promoter strength.

The nucleotide sequences of SUC2 promoters from strains BY4741, NCYC625 and L610 were blasted using DNAStar MegAlign program. As shown in Figure 4B, there were three nucleotide substitutions in promoter sequence of strain L610 when compared with that of strains BY4741 and NCYC625.

Those three promoters were used to drive the expression of SUC2 separately in strain BY4741. As shown in 


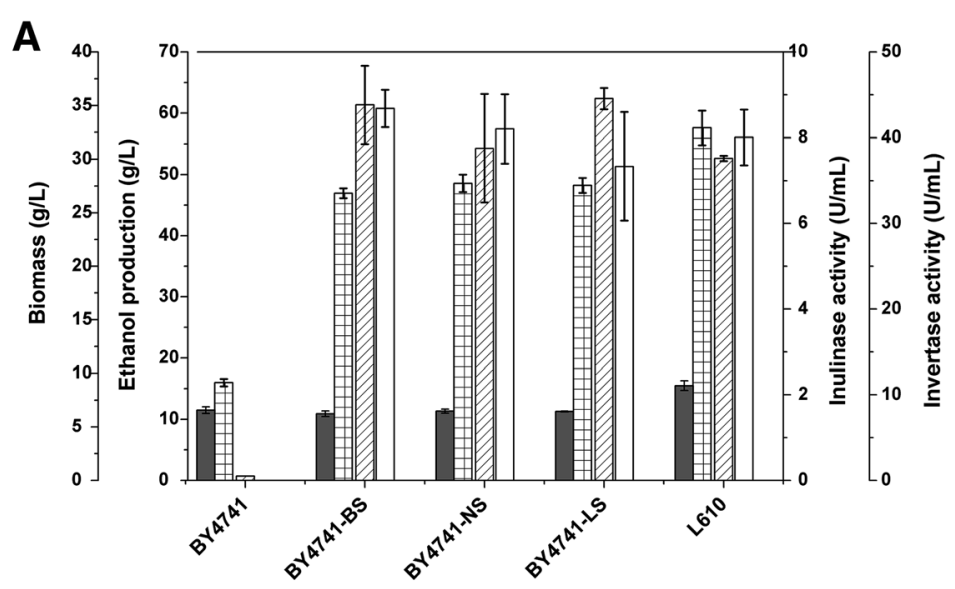

B

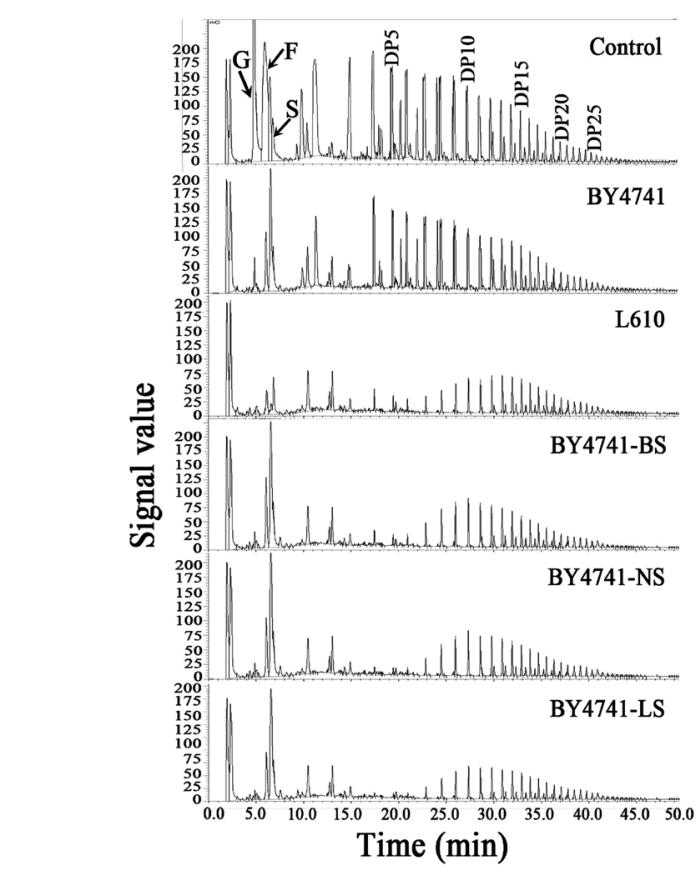

C

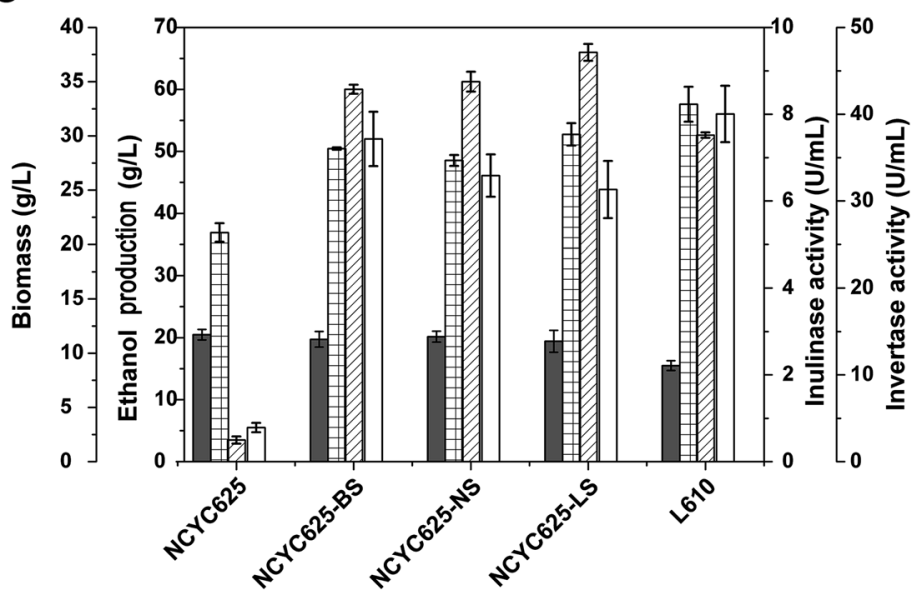

Figure 3 (See legend on next page.) 
(See figure on previous page.)

Figure $\mathbf{3}$ Inulin-fermenting performances of weak-inulin-utilizing yeast strains overexpressed with SUC2. (A) Biomass (dry cell weight, gray), ethanol production (grid), inulinase activity (diagonal) and invertase activity (blank) of strains BY4741 overexpressing different SUC2 versions were assayed after cultured in inulin medium at $30^{\circ} \mathrm{C}$ for $48 \mathrm{~h}$, in which BS, NS and LS indicated different SUC2 versions from strains BY4741, NCYC625 and $\mathrm{L} 610$ respectively and error bars represent standard deviations from the mean of three biological samples; (B) Analysis of the inulin hydrolysate in the culture supernatant by ion chromatography, in which the supernatant of the 0-h culture was used as a control and all strains were cultivated in inulin medium for 48 h. Abbreviation G, F, and S represent glucose, fructose, and sucrose, respectively; (C) Biomass (dry cell weight, gray), ethanol production (grid), inulinase activity (diagonal) and invertase activity (blank) of strains NCYC625 overexpressing different SUC2 versions were assayed, in which the culture condition, the indication of BS, NS and LS and the representation of error bars are the same as that of BY4741 recombinants.

Figure 4C, SUC2 expression under the control of the promoter from strain BY4741 (BPS) showed low ethanol production $(26 \mathrm{~g} / \mathrm{L})$, inulinase $(1.5 \mathrm{U} / \mathrm{mL})$ and invertase activity $(0.2 \mathrm{U} / \mathrm{mL})$. Low ethanol production $(25 \mathrm{~g} / \mathrm{L})$, inulinase $(1.1 \mathrm{U} / \mathrm{mL})$ and invertase activity $(0.3 \mathrm{U} / \mathrm{mL})$ were also obtained when SUC2 expression was driven by the promoter from strain NCYC625 (NPS). However, the SUC2 expression under the control of the promoter from strain L610 (LPS) showed high ethanol yield (44 g/L), inulinase activity $(6.7 \mathrm{U} / \mathrm{mL})$ and invertase activity $(42.6 \mathrm{U} / \mathrm{mL})$. Those results indicated that the promoter strength in driving the expression of SUC2 determined the inulindegrading capability of strains BY4741, NCYC625 and L610.

\section{Other factors have a minor effect on inulin utilization}

To elucidate whether $S$. cerevisiae strain L610 carries other genes or trans-acting factors responsible for inulin fermentation, haploid strain L610 $\alpha$ and its mutant L610 $\alpha$ suc $2 \Delta$ were prepared and mated with strain BY4741 and mutant BY4741 suc2 $\Delta$, respectively. Four different heterozygotes were obtained, including L610 $\alpha \times$ BY4741 (LB11), $\mathrm{L} 610 \alpha \times$ BY4741 suc $2 \Delta$ (LB12), L610 $\alpha$ suc $2 \Delta \times$ BY4741 (LB13) and L610 $\alpha$ suc2 $\Delta \times$ BY4741 suc2 $\Delta$ (LB14). No inulinase activity could be detected in strains $\mathrm{L} 610 \alpha$ suc $2 \Delta$ and BY4741 suc $2 \Delta$ (data not shown). As shown in Figure 5, the inulinase activity in heterozygote LB11 was comparable with that in LB12 whose SUC2 allele from BY4741was deleted. Whereas trace-level inulinase activity was observed in strain LB13 when SUC2 allele was deleted only from L610 $\alpha$, no enzyme activity remained in heterozygote LB14 when both SUC2 alleles were deleted.

\section{Discussion}

Invertase Suc2 (EC 3.2.1.26) belongs to sucrosehydrolyzing enzyme but plays a key role in inulin catabolism by catalyzing inulin hydrolysis in yeast $S$. cerevisiae [10]. However, the inulinase activity of Suc2 can be detected only in some $S$. cerevisiae strains although SUC2 is a constitutive genomic component [16]. As shown in Figure 1, all strains L610, NCYC625 and BY4741 could grow well in the inulin medium, whereas the Suc2 activity and ethanol production in strain L610 is much higher than that in strains NCYC625 and BY4741. It is presumed that biomass and ethanol production of strains
NCYC625 and BY4741 is mainly from the residual glucose and fructose in the inulin medium but not from inulin (Figure 3B). In fact, a little inulin was catabolised by strain BY4741 compared with strain L610 after incubation in inulin medium for $48 \mathrm{~h}$ (Figure 3B). Therefore, both strains NCYC625 and BY4741 could weakly catabolize inulin and strain L610 could strongly utilize inulin for ethanol production.

It has been reported that SUC2 gene encodes two different forms of invertase, external and internal enzyme. Internal invertase has no function in sucrose hydrolyzation, whereas the external invertase is excreted in the periplasmic space and plays the main role in sucrose hydrolysis $[17,18]$. It has been showed that the extracellular Suc2 is a glycosylated homodimer containing 14 potential N-linked glycosylation sites, in which eight sites are completely glycosylated and five sites are partially glycosylated $[13,19]$. Blast result of Suc2 sequences from S. cerevisiae strains BY4741, NCYC625, JZ1C and L610 showed five variations, including N84H, Q88E, A409P (BS vs. NS, JS, LS), V431A (BS, NS vs. JS, LS) and I247S (NS vs. BS, JS, LS). However, no variation in amino acid sequences was included in the glycosylation site (Figure 2). Therefore, the discrepancy in the inulinase activity should not be attributed to the glycosylation of invertase Suc 2 .

As previously reported [14,15,20-22], several conserved domains (underlined) and residues (Trp39, His56, Phe102, Asp171, Glu223 and Cys224) were predicted to be important for either substrate binding or catalysis (Figure 2). However, no variation in amino acid sequences in $S$. cerevisiae strains was located in those critical sites, indicating that the sequence changes in Suc2 were not the main reason for the discrepancy in enzyme activity.

It is significant that the overexpression of SUC2 versions (LS, NS and BS) in strains BY4741 or NCYC625 makes the inulin-fermenting ability increase from zero to hero. The increased ethanol production from inulin with high degree of polymerization (around 48-53 g/L at $24 \mathrm{~h}$ ) was obtained by all the SUC2 overexpressing strains, which were higher than that by other inulinfermenting wild $S$. cerevisiae strains, such as strain KCCM50549 (36.2 $\mathrm{g} / \mathrm{L}$ at $34 \mathrm{~h})$ [7]. The increased 

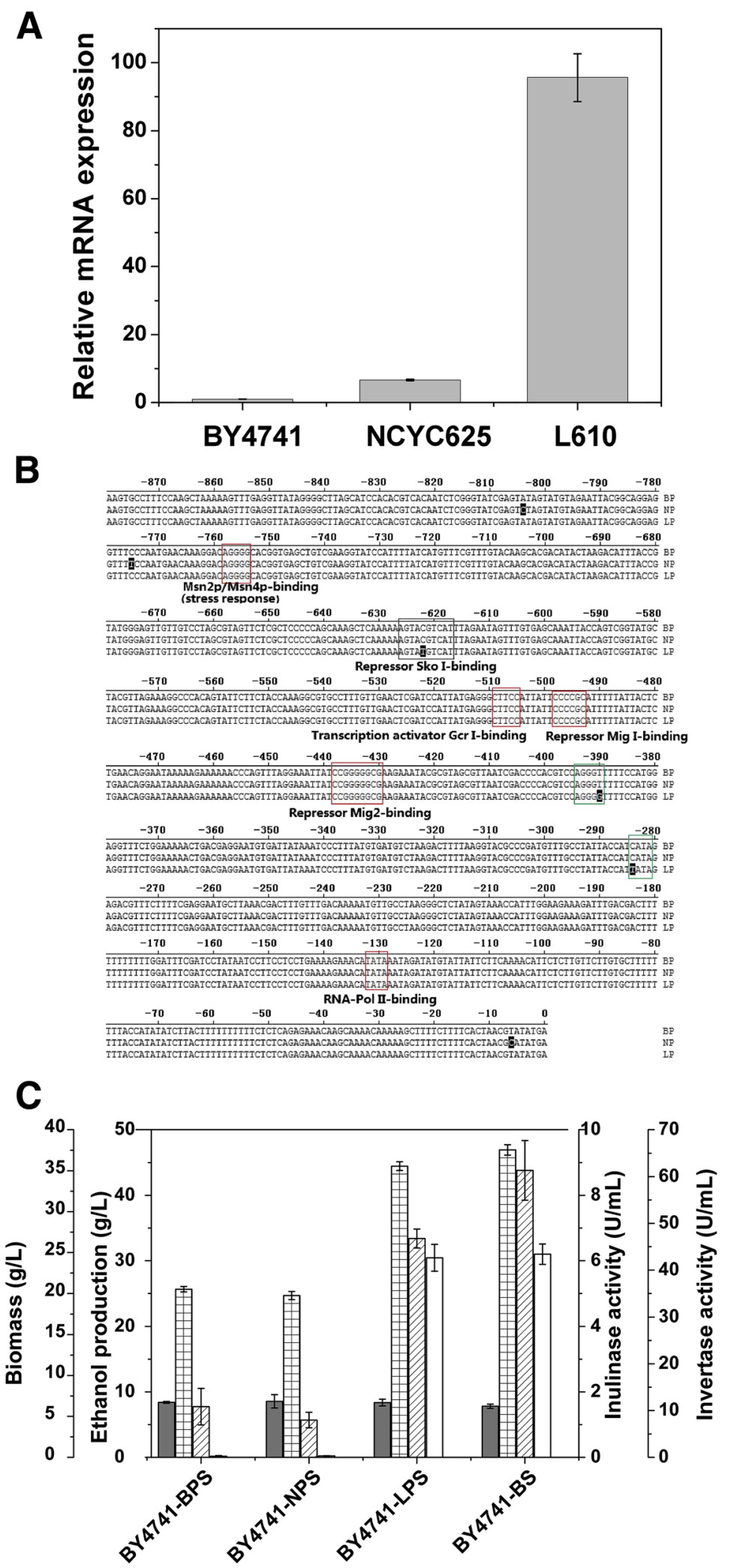

Figure 4 (See legend on next page.) 
(See figure on previous page.)

Figure 4 Effect of SNPS in the SUC2 promoter on SUC2 transcript level. (A) SUC2 mRNA levels in S. cerevisiae strains BY4741, NCYC625 and L610 were determined by qRT-PCR, in which the SUC2 transcription level in strain BY4741 was set as 1, while others in strains NCYC625 and L610 were compared with it and showed as relative data; (B) Sequence alignment of SUC2 promoters from BY4741 (BP), NCYC625 (NP) and L610 (LP) by CLUSTAL W program. Important transcription factor binding sites were boxed in red. Nucleotide substitutions among three strains were blacked. A novel AGGGG sequence and a new TATA box formed by two nucleotide substitutions in SUC2 promoter of stain L610 were boxed in green; (C) Biomass (dry cell weight, gray), ethanol production (grid), inulinase activity (diagonal) and invertase activity (blank) of strain BY4741 overexpressing SUC2 under the control of different strength promoters were measured after cultured in inulin medium at $30^{\circ} \mathrm{C}$ for $48 \mathrm{~h}$. Abbreviation BPS, NPS and LPS indicated SUC2 expression under the control of the promoter from strain BY4741, NY4741 and L610 respectively, and BS indicated SUC2 version from strain BY4741 under the control of the constitutive PGK1 promoter. Error bars represent standard deviations from the mean of three biological samples.

inulinase activity (around $8 \mathrm{U} / \mathrm{mL}$ ) and invertase activity (31-43 U/mL) in recombinants was well correlated with the raised ethanol yield, which was much higher than that in strain JZ1C (around $1.5 \mathrm{U} / \mathrm{mL}$ for inulinase and $18 \mathrm{U} / \mathrm{mL}$ for invertase) [10]. All those results implied that the expression level of $S U C 2$ was probably the key factor for inulin catabolism in S. cerevisiae. Such speculation was confirmed by qRT-PCR results, which showed a progressive increase in SUC2 transcript levels in order of strains BY4741, NCYC625 and L610 (Figure 4A).

The influence of promoter sequence variation on the expression level of SUC2 was investigated. Several transcription factor binding sites have been reported to be located within the SUC2 promoter, including TATA box, elements of catabolite repression (Mig1/Mig2 and Sko1 binding sequence) and transcriptional activation (Gcr1, Msn2p/Msn4p binding sites) [23-27]. As shown in Figure 4B, there are three nucleotide substitutions in the SUC2 promoter of strain L610 when compared with that of strains BY4741 and NCYC625. The first nucleotide substitution in SUC2 promoter of strain L610 was located in the position -627 to -617 . Such mutation presumably resulted in the derepression of SUC2 because the region between -627 and -617 acts as a negative element and can be recognized by repressor Sko1. The second nucleotide substitution in SUC2 promoter of stain L610 formed a novel AGGGG (Msn2p/Msn4p binding) sequence in the position -390 to -394 , which was called as STRE (stress response elements) and considered to be able to mediate transcription induced by environmental stress. The possible function of the third substitution in SUC2 promoter of strain L610 might be related to the formation of a TATA box in position -281 to -284 . As for the fermentation results, Suc2 in strain BY4741 under the control of the L610 SUC2 promoter showed much higher enzyme activity than that under the control of other two promoters, which was in accordance with the presumption of promoter mutation. All those results suggested that the expression level of SUC2 in S. cerevisiae strains was affected by the sequence variation in SUC2 promoters, leading to different capability of inulin catabolism.

In order to confirm whether Suc2 is the only inulindegrading enzyme in strain L610 and if there are any other elements responsible for inulin fermentation except the promoter, gene knock out and mating experiments were performed. No inulinase activity could be detected when SUC2 gene in haploid strain L610 $\alpha$ was deleted, suggesting that Suc2 is the key enzyme for inulin degradation in natural $S$. cerevisiae strains. When compared with strain BY4741 or heterozygote strain LB14, some inulinase activity was recovered in strain LB13, suggested that some trans-acting factors from L610 $\alpha$ might have a weak interaction with cis-elements of SUC2 allele from BY4741. Therefore, other elements had a minor effect on inulinase activity of Suc2 in addition to the SUC2 promoter.

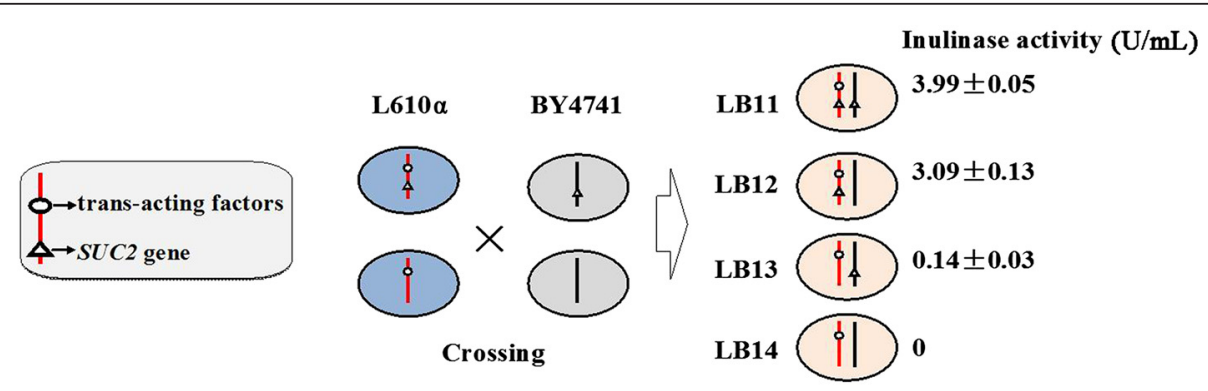

Figure 5 SUC2/suc2 heterozygote construction and the inulinase activity. Mating experiment was performed between haploid strains L610a (blue) and BY4741 (gray) with or without SUC2. Four different heterozygotes, including L610a XBY4741 (LB11), L610a $\times$ BY4741 suC2 $\triangle$ (LB12), $L 610$ a suc2 $\triangle \times B Y 4741$ (LB13) and L610a suc2 $\triangle \times B Y 4741$ suc2 $\triangle$ (LB14), were produced. The inulinase activities were expressed as means \pm standard deviation. 


\section{Conclusions}

The transcription level of SUC2 is the key factor affecting the inulinase activity and inulin catabolism ability of $S$. cerevisiae strains. The sequence variation in SUC2 promoter resulted in different transcript level of $S U C 2$ in different S. cerevisiae strains.

\section{Methods}

\section{Strains and culture condition}

S. cerevisiae BY4741 was a kind gift from Prof. Zhao (Dalian Institute of Chemical Physics, CAS). S. cerevisiae NCYC625 was purchased from China General Microbiological Culture Collection Centre. S. cerevisiae L610 was isolated as described previously [28]. Other information for S. cerevisiae strains used in this study was listed in Table 1. Escherichia coli DH5 $\alpha$ was used in all cloning experiments.

S. cerevisiae BY4741, NCYC625 and their recombinant strains were routinely cultivated at $30^{\circ} \mathrm{C}$ in YPD medium $(20 \mathrm{~g} / \mathrm{L}$ glucose, $10 \mathrm{~g} / \mathrm{L}$ yeast extract and $20 \mathrm{~g} / \mathrm{L}$ peptone, $\mathrm{pH}$ 6.0) supplemented with $200 \mathrm{mg} / \mathrm{L}$ G418 if necessary. E. coli $\mathrm{DH} 5 \alpha$ was grown at $37^{\circ} \mathrm{C}$ in Luria-Bertani (LB) medium $(10 \mathrm{~g} / \mathrm{L}$ tryptone, $5.0 \mathrm{~g} / \mathrm{L}$ yeast extract, and $10 \mathrm{~g} / \mathrm{L}$ sodium chloride, $\mathrm{pH}$ 7.0) supplemented with $100 \mathrm{mg} / \mathrm{L}$ ampicillin if necessary.

\section{Cloning of SUC2 genes and promoters}

The full-length SUC2 genes and their corresponding promoters were amplified from the genomic DNA of $S$. cerevisiae strains BY4741, NCYC625 and L610 respectively, using PrimeSTAR HS DNA Polymerase (TaKaRa Bio Inc., Dalian, China) and primers listed in Additional file 1: Table S1. The PCR products were ligated into plasmid pMD18-T (TaKaRa Bio Inc., Dalian, China) and sequenced.

Alignment of multiple nucleotide/amino acid sequences was performed using the CLUSTAL W program [29].

\section{Plasmid construction and yeast transformation}

For overexpression assay in strains BY4741 and NCYC625, SUC2 gene of strain BY4741 in the plasmid pYC230-BSUC2 (a kind gift from Fu-Li LI, Qingdao Institute of BioEnergy and Bioprocess Technology, CAS) was replaced by the SUC2 gene of strain NCYC625 or strain L610 using a restriction-free (RF) cloning method described before [30]. To observe the effect of promoter on SUC2 transcription, the PGK1 promoter in the plasmid pYC230-B-SUC2 was replaced by the $S U C 2$ promoters in strain BY4741, NCYC625 or L610, respectively, using RF cloning method. All primers used for plasmid construction were listed in Additional file 1: Table S1. All plasmids were confirmed by restriction enzyme digestion and

Table 1 Strains used in this study

\begin{tabular}{|c|c|c|}
\hline Strain & Genotype & Source or reference \\
\hline BY4741 & MATa his $3 \Delta 1$ leu2 $\triangle 0$ met15 $\triangle 0$ ura $3 \Delta 0$ & A gift from Prof. Zhao Z \\
\hline BY4741-BS & BY4741 containing pYC230-B-SUC2 ${ }^{\mathrm{a}}$ & This study \\
\hline BY4741-NS & BY4741 containing pYC230-N-SUC2 ${ }^{\mathrm{b}}$ & This study \\
\hline BY4741-LS & BY4741 containing pYC230-L-SUC2 ${ }^{C}$ & This study \\
\hline BY4741-BPS & BY4741 containing pYC230-BP-BSUC2 ${ }^{d}$ & This study \\
\hline BY4741-NPS & BY4741 containing pYC230-NP-BSUC2 & This study \\
\hline BY4741-LPS & BY4741 containing pYC230-LP-BSUC2 & This study \\
\hline NCYC625 & Wild type, diploid & CGMCC \\
\hline NCYC625-BS & NCYC625 containing pYC230-BSUC2 & This study \\
\hline NCYC625-NS & NCYC625 containing pYC230-NSUC2 & This study \\
\hline NCYC625-LS & NCYC625 containing pYC230-LSUC2 & This study \\
\hline L610 & Wild type, diploid & Yang et al. \\
\hline L610a & Haploid, MATa & This study \\
\hline L610a suc2 $\triangle$ & L610a in which SUC2 gene was deleted & This study \\
\hline BY4741 suc2 $\triangle$ & BY4741 in which SUC2 gene was deleted & This study \\
\hline LB11 & Heterozygote: L610a ×BY4741 & This study \\
\hline LB12 & Heterozygote: L610a $\times$ BY4741 suc2 $\triangle$ & This study \\
\hline LB13 & Heterozygote: L610a suc2 $\triangle \times B Y 4741$ & This study \\
\hline LB14 & Heterozygote: L610a suc2 $\triangle \times$ BY4741 suc2 $\triangle$ & This study \\
\hline
\end{tabular}

${ }^{a}$ B-SUC2 indicates the SUC2 gene of BY4741;

${ }^{\mathrm{b}} \mathrm{N}$ indicates the strain NCYC625;

${ }^{\mathrm{C}} \mathrm{L}$ indicates the strain $\mathrm{L} 610$;

${ }^{\mathrm{d}} \mathrm{BP}$ indicates PGK1 promoter in the plasmid pYC230-B-SUC2 was replaced by the promoter of BY4741 SUC2. 
sequencing. All constructs were transformed into $S$. cerevisiae strains according to the electroporation protocol [31].

\section{SUC2 expression analysis}

RNA was extracted from S. cerevisiae strain BY4741, NCYC625 and L610 respectively using RNAiso Plus according to manufacturer's instruction (TaKaRa Bio Inc.). Totally 100 ng RNA was reverse transcribed using PrimeScript RT reagent Kit with gDNA Eraser (TaKaRa Bio Inc.). The reverse transcription product was diluted to one-tenth and used as the template for quantitative real-time PCR (qRT-PCR) with TaKaRa PCR Thermal Cycler Dice Real Time System (TaKaRa Code.TP800). To normalize the cycle threshold values, the relative transcript level for the housekeeping gene ACT1 was determined. The condition used for qRT-PCR was as follows: $95^{\circ} \mathrm{C}$ for $30 \mathrm{~s}, 40$ cycles of $95^{\circ} \mathrm{C}$ for $5 \mathrm{~s}, 60^{\circ} \mathrm{C}$ for $30 \mathrm{~s}$ [32]. The primers used for qRT-PCR were listed in Additional file 1: Table S1.

\section{SUC2/suc2 heterozygote construction}

The haploid S. cerevisiae L610 strains were prepared by sporulation and tetrad dissection as described before [33]. The expected 2:2 pattern of L610a (MATa) and L610 $\alpha$ (MAT $\alpha)$ was identified by colony PCR, and primer pairs MAT-F/MAT-a or MAT-F/MAT- $\alpha$ (listed in Additional file 1: Table S1) were used. The condition for colony PCR was as follows: $95^{\circ} \mathrm{C}$ for $10 \mathrm{~min}, 30$ cycles of $95^{\circ} \mathrm{C}$ for $45 \mathrm{~s}, 52^{\circ} \mathrm{C}$ for $40 \mathrm{~s}, 72^{\circ} \mathrm{C} 45 \mathrm{~s}$.

The SUC2 gene in haploid L610 $\alpha$ and BY4741 (MATa) strains was deleted using a PCR-mediated gene disruption method based on homologous recombination as reported by Wang et al. [10], resulting in $\mathrm{L} 610 \alpha$ suc2 $\Delta$ and BY4741 suc $2 \Delta$ mutants.

Mating experiment was performed according to the classical protocol [33]. In brief, single colonies of L610a and BY4741 with or without SUC2 gene were mixed and incubated in fresh YPD for $4 \mathrm{~h}$. The mixed culture was diluted and plated on an YPD agar plate and cultivated at $30^{\circ} \mathrm{C}$. The hybridized diploid strains were identified by colony PCR using the primers MAT-F/MAT-a/MAT- $\alpha$ together.

\section{Ethanol production}

After incubated in $50 \mathrm{~mL}$ YPD medium supplemented with $100 \mathrm{mg} / \mathrm{L}$ G418 if necessary at $30^{\circ} \mathrm{C}$ and $150 \mathrm{rev} /$ min for $24 \mathrm{~h}$, the culture of S. cerevisiae strains was inoculated in $50 \mathrm{~mL}$ inulin medium with an inoculum of $5 \%(\mathrm{v} / \mathrm{v})$ and cultivated at $30^{\circ} \mathrm{C}$ for $48 \mathrm{~h}$ for ethanol production. Inulin medium contained $150 \mathrm{~g} / \mathrm{L}$ inulin, $5 \mathrm{~g} / \mathrm{L}$ yeast extract and $0.5 \mathrm{~g} / \mathrm{L} \mathrm{MgSO}_{4}$ at $\mathrm{pH}$ 6.0. The culture was centrifuged at $4^{\circ} \mathrm{C}$ and $8000 \mathrm{~g}$ for $10 \mathrm{~min}$ and the supernatant was ready for measuring ethanol concentration and inulin degradation.

\section{Analysis of biomass and ethanol concentration}

Biomass was determined by measuring the cell dry weight, which was obtained from cell pellet in $50 \mathrm{~mL}$ of culture broth, and dried in an oven at $105^{\circ} \mathrm{C}$ to a constant weight.

Ethanol concentration in the culture supernatant was measured by gas chromatography (Agilent Technologies Inc.) and both data acquisition and treatment were realized by the software Agilent ChemStation as described before [28].

\section{Enzyme assay}

Inulinase or invertase activity was measured as described previously $[10,34]$. The reaction mixture consists of $50 \mu \mathrm{L}$ cell culture and $450 \mu \mathrm{L}$ of $2 \%$ inulin or sucrose in $100 \mathrm{mM}$ acetate buffer ( $\mathrm{pH}$ 5.0). The reaction was performed at $50^{\circ} \mathrm{C}$ for $15 \mathrm{~min}$ and terminated by boiling. The reducing sugar was assayed by the 3,5-dinitrosalicylic acid method [35]. One unit of enzyme activity was defined as the amount of enzyme that produced $1 \mu \mathrm{mol}$ fructose per min under the assay condition used in this study.

\section{Ion chromatography}

The degree of polymerization of inulin-type oligosaccharide in the culture supernatant before and after inulin fermentation by $S$. cerevisiae was analyzed by ion chromatography (Thermo Fisher Inc., ICS-5000) equipped with cation exchange analytical column Carbo Pac PA200 (250 $\mathrm{mm} \times 3 \mathrm{~mm})$ and cation exchange guard column Carbo Pac PA200 (50 mm $\times 3 \mathrm{~mm})$. The column temperature was set at $30^{\circ} \mathrm{C}$ at a flow rate of $0.5 \mathrm{~mL} /$ $\mathrm{min}$. The culture supernatant was diluted 100 -fold and a volume of $25 \mu \mathrm{L}$ of sample was injected. The oligosaccharides were separated by elution with $200 \mathrm{mM} \mathrm{NaOH}$ and $1 \mathrm{M} \mathrm{NaOAc}$.

\section{Statistical analysis}

All of the assays and determinations described in this paper were performed in triplicate unless otherwise stated. Data was expressed as the means of three biological samples \pm standard deviation.

\section{Additional file}

Additional file 1: Table S1. Primers used in this study. All the primers were synthesized by TaKaRa Bio Inc.

\section{Competing interests}

The authors declare that they have no competing interests. 


\section{Authors' contributions}

FY designed and participated in the experiment and data analysis as well as drafting the manuscript. ZCL and XW carried out molecular genetic study and fermentation experiment. LLL participated in the GRT-PCR and fermenting experiment. LY contributed to the sequence alignment and the data analysis. WZT participated in the design and performed the statistical analysis. ZMY participated in ion chromatography experiment. $X \mathrm{~L}$ conceived the study, supervised and contributed to final drafting of the manuscript. All authors read and approved the final manuscript.

\section{Acknowledgements}

We would like to thank Z Zhao and F Li for providing strain and plasmid. We also appreciate Dr. Yongjin Zhou (Chalmers University of Technology) for reading the manuscript and providing many valuable comments. Financial supports provided by Natural Sciences Foundation of China (31201302, 31371742), Special Fund for Agroscientific (marine) Research in the Public Interest (201303095, 201405003), Science and technology Department of Dalian (2013B11NC078 and 2012J21DW009), Education Department of Liaoning (L2012197) are greatly acknowledged.

\section{Received: 11 July 2014 Accepted: 8 April 2015}

\section{Published online: 17 April 2015}

\section{References}

1. Chi ZM, Zhang T, Cao TS, Liu XY, Cui W, Zhao CH. Biotechnological potential of inulin for bioprocesses. Bioresoure Technol. 2011;102:4295-303.

2. Bajpai PK, Bajpai P. Cultivation and utilization of Jerusalem artichoke for ethanol, single cell protein, and high-fructose syrup production. Enzyme Microb Technol. 1991;13:359-62.

3. Negro MJ, Ballesteros I, Manzanares P, Oliva JM, Sáez F, Ballesteros M. Inulin-containing biomass for ethanol production: carbohydrate extraction and ethanol fermentation. Appl Biochem Biotechnol. 2006;129-132:922-32.

4. Yuan W, Zhao X, Ge X, Bai F. Ethanol fermentation with Kluyveromyces marxianus from Jerusalem artichoke grown in salina and irrigated with a mixture of seawater and freshwater. J Appl Microbiol. 2008;105:2076-83.

5. Lane MM, Morrissey JP. Kluyveromyces marxianus: A yeast emerging from its sister's shadow. Fungal Biol Rev. 2010;24:17-26.

6. Ohta K, Hamada S, Nakamura T. Production of high concentrations of ethanol from inulin by simultaneous saccharification and fermentation using Aspergillus niger and Saccharomyces cerevisiae. Appl Environ Microbiol. 1993;59:729-33.

7. Lim S-H, Ryu J-M, Lee H, Jeon JH, Sok D-E, Choi E-S. Ethanol fermentation from Jerusalem artichoke powder using Saccharomyces cerevisiae KCCM50549 without pretreatment for inulin hydrolysis. Bioresoure Technol. 2011;102:2109-11.

8. Schorr-Galindo S, Ghommidh C, Guiraud J. Influence of yeast flocculation on the rate of Jerusalem artichoke extract fermentation. Curr Microbiol. 2000;41:89-95.

9. Yuan B, Wang SA, Li FL. Improved ethanol fermentation by heterologous endoinulinase and inherent invertase from inulin by Saccharomyces cerevisiae. Bioresoure Technol. 2013;139:402-5.

10. Wang SA, Li FL. Invertase Suc2 Is the key hydrolase for inulin degradation in Saccharomyces cerevisiae. Appl Environ Microbiol. 2013;79:403-6.

11. Madsen KM, Udatha GD, Semba S, Otero JM, Koetter P, Nielsen J, et al. Linking genotype and phenotype of Saccharomyces cerevisiae strains reveals metabolic engineering targets and leads to triterpene hyper-producers. PLoS One. 2011;6:e14763.

12. Alper H, Fischer C, Nevoigt E, Stephanopoulos G. Tuning genetic control through promoter engineering. Proc Natl Acad Sci U S A. 2005;102:12678-83.

13. Reddy V, Johnson R, Biemann K, Williams R, Ziegler F, Trimble R, et al. Characterization of the glycosylation sites in yeast external invertase. I. $\mathrm{N}$-linked oligosaccharide content of the individual sequons. J Biol Chem. 1988;263:6978-85.

14. Ohta K, Akimoto H, Matsuda S, Toshimitsu D, Nakamura T. Molecular cloning and sequence analysis of two endoinulinase genes from Aspergillus niger. Biosci Biotechnol Biochem. 1998;62:1731-8.

15. Lafraya Á, Sanz-Aparicio J, Polaina J, Marín-Navarro J. Fructo-oligosaccharide synthesis by mutant versions of Saccharomyces cerevisiae invertase. Appl Environ Microbiol. 2011;77:6148-57.
16. Yuji Oda DM, Leo F, Urashima T. Discrimination of Saccharomyces cerevisiae and Saccharomyces paradoxus strains by the SUC2 gene sequences. J Gen Appl Microbiol. 2010;56:355-8.

17. Gascón S, Neumann NP, Lampen JO. Comparative study of the properties of the purified internal and external invertases from yeast. J Biol Chem. 1968;243:1573-7.

18. Trimble RB, Maley F. Subunit structure of external invertase from Saccharomyces cerevisiae. J Biol Chem. 1977;252:4409-12.

19. Taussig R, Carlson M. Nucleotide sequence of the yeast SUC2 gene for invertase. Nucleic Acids Res. 1983;11:1943-54.

20. Sainz-Polo MA, Ramírez-Escudero M, Lafraya A, González B, Marín-Navarro J, Polaina J, et al. Three-dimensional structure of Saccharomyces invertase: role of a non-catalytic domain in oligomerization and substrate specificity. J Biol Chem. 2013;288:9755-66

21. Park J, Kim MI, Park YD, Shin I, Cha J, Kim CH, et al. Structural and functional basis for substrate specificity and catalysis of levan fructotransferase. J Biol Chem. 2012;287:31233-41.

22. Pons T, Olmea O, Chinea G, Beldarraín A, Márquez G, Acosta N, et al. Structural model for family 32 of glycosyl-hydrolase enzymes. Proteins. 1998;33:383-95.

23. Wu L, Winston F. Evidence that Snf-Swi controls chromatin structure over both the TATA and UAS regions of the SUC2 promoter in Saccharomyces cerevisiae. Nucleic Acids Res. 1997;25:4230-4.

24. Martinez-Pastor M, Marchler G, Schüller C, Marchler-Bauer A, Ruis H, Estruch $F$. The Saccharomyces cerevisiae zinc finger proteins Msn2p and Msn4p are required for transcriptional induction through the stress response element (STRE). EMBO J. 1996;15:2227-35.

25. Bu Y, Schmidt MC. Identification of cis-acting elements in the SUC2 promoter of Saccharomyces cerevisiae required for activation of transcription. Nucleic Acids Res. 1998;26:1002-9.

26. Weinhandl K, Winkler M, Glieder A, Camattari A. Carbon source dependent promoters in yeasts. Microb Cell Fact. 2014; 13: doi:10.1186/1475-2859-13-5.

27. Lutfiyya $L L$, Johnston $M$. Two zinc-finger-containing repressors are responsible for glucose repression of SUC2 expression. Mol Cell Biol. 1996;16:4790-7.

28. Yang F, Liu Z, Dong W, Zhu L, Chen X, Li X. Ethanol production using a newly isolated Saccharomyces cerevisiae strain directly assimilating intact inulin with high degree of polymerization. Biotechnol Appl Biochem. 2013;61:418-25.

29. Thompson JD, Higgins DG, Gibson TJ. CLUSTAL W: improving the sensitivity of progressive multiple sequence alignment through sequence weighting, position-specific gap penalties and weight matrix choice. J Nucleic Acids Res. 1994;22:4673-80.

30. Van Den Ent F, Löwe J. RF cloning: a restriction-free method for inserting target genes into plasmids. J Biochem Biophys Methods. 2006;67:67-74.

31. Thompson JR, Register E, Curotto J, Kurtz M, Kelly R. An improved protocol for the preparation of yeast cells for transformation by electroporation. Yeast. 1998;14:565-71.

32. Stahlberg A, Elbing K, Andrade-Garda JM, Sjogreen B, Forootan A, Kubista M. Multiway real-time PCR gene expression profiling in yeast Saccharomyces cerevisiae reveals altered transcriptional response of $\mathrm{ADH}$-genes to glucose stimuli. BMC Genomics. 2008; 9: doi:10.1186/1471-2164-9-170.

33. Adams A, Gottschling D-E, Kaiser C-A, Stearns T. Methods in yeast genetics - a cold spring harbor laboratory course manual. Cold Spring Harbor, NY: Cold Spring Harbor Laboratory Press; 1997.

34. Hu N, Yuan B, Sun J, Wang S-A, Li F-L. Thermotolerant Kluyveromyces marxianus and Saccharomyces cerevisiae strains representing potentials for bioethano production from Jerusalem artichoke by consolidated bioprocessing. Appl Microbiol Biotechnol. 2012;95:1359-68.

35. Miller GL. Use of dinitrosalicylic acid reagent for determination of reducing sugar. Anal Chem. 1959;31:426-8. 